\title{
Editorial
}

\section{Warfarin in atrial fibrillation: underused in the elderly, often inappropriately used in the young}

In the past decade, much has been learned about antithrombotic prophylaxis to prevent stroke in patients with non-valvar atrial fibrillation (AF). Adjusted dose warfarin is highly efficacious, aspirin is modestly efficacious (reducing primarily non-disabling, non-cardioembolic strokes in $\mathrm{AF}$ ), warfarin is much more efficacious than aspirin, and low intensity warfarin (international normalised ratios (INR) < 1.5) alone or combined with aspirin offers minimal protection. ${ }^{1}$ The absolute risk of stroke varies widely in patients with $\mathrm{AF}$, from $<1 \%$ to $12 \%$ per year, depending on age and coexisting vascular disease. ${ }^{2}{ }^{3}$ Selection of antithrombotic prophylaxis should consider the inherent risk of stroke and the absolute risk reductions afforded. It is my view that many AF patients, including most younger than 75 years, do not substantially benefit from treatment with adjusted dose warfarin.

Two observational studies in this issue address this important, controversial issue. ${ }^{4}{ }^{5}$ Perez and colleagues surveyed a sample of patients with AF randomly drawn from outpatient clinics. ${ }^{4}$ Applying a scheme for stratifying stroke risk, only about half of those deemed high risk and who had no apparent contraindications for anticoagulation were being treated with warfarin, while a quarter of those presumed at low risk were receiving anticoagulants. Warfarin was particularly underused among high risk $\mathrm{AF}$ patients older than 75 years: only about $20 \%$ were being anticoagulated. The second study by Deplanque and the SAFE I (stroke in atrial fibrillation ensemble) Study Investigators examined the use of antithrombotic agents before hospitalisation among $213 \mathrm{AF}$ patients with acute stroke or transient ischaemic attack. ${ }^{5}$ Their results suggest considerable room for improvement but are more difficult to interpret as the risk of stroke in those not given antithrombotic agents in the population from which these cases were drawn cannot be estimated, and risk stratification was not examined. The mean age of outpatients with AF in Perez et al's study was 68 years, ${ }^{4}$ while the mean age of AF patients with cerebral ischaemia was nearly a decade older in the SAFE I study. ${ }^{5}$ Both studies found advancing age to be inversely correlated with warfarin use. ${ }^{45}$

\section{Underuse of warfarin in patients with AF older than 75 years}

About $50 \%$ of people with non-valvar AF in population based surveys are $>75$ years, and the bulk of AF associated stroke occurs in this age group. The risk of major bleeding during anticoagulation, including devastating intracranial haemorrhage, is higher in the very elderly, ${ }^{6}$ but the risks do not offset the substantial benefits for most high risk $\mathrm{AF}$ patients if anticoagulants are carefully administered. ${ }^{27}$ While INRs between 2 and 3 appear to provide optimal protection against stroke for most AF patients, ${ }^{8}$ targeting the lower end of this range may be sensible for primary prevention in those $>75$ years to minimise major and minor haemorrhage, the latter frequently leading to discontinuation of warfarin. ${ }^{9}$ More studies are needed on the safety of anticoagulation and tolerance over time for AF patients $>75$ years treated in clinical practice. Nevertheless, it seems clear that warfarin is underused in elderly, high risk AF patients, neglecting an important opportunity for stroke prevention. ${ }^{10}$

\section{Predicting stroke risk in AF patients}

Warfarin reduces stroke for all patients with $\mathrm{AF}$, but the magnitude of reduction is small for the many patients with low inherent risks for stroke (table 1). The number of AF patients who would need to be treated with warfarin instead of aspirin for one year to prevent one stroke (the number needed to treat) is about 14 for high risk AF patients with recent cerebral ischaemia compared with nearly 250 for AF patients at low risk for stroke.

Can stroke risk in AF patients be reliably predicted? Four schemes have been generated by multivariate analyses of prospectively studied clinical trial cohorts (table 2). ${ }^{11-14}$ While criteria overlap, the differences importantly influence patient management. Depending on which scheme is used, the fraction of an AF cohort categorised as high risk can vary from $33-88 \% .{ }^{15}$ Most AF patients over age 75 years $(66-100 \%)$ are predicted to be high risk compared with as few as $6 \%$ of younger $\mathrm{AF}$ patients without prior stroke, depending on the criteria. ${ }^{13}$ In one scheme, results of precordial echocardiography did not contribute to risk stratification, ${ }^{13}$ although this remains controversial. ${ }^{10}$ Whether transoesophageal echocardiography contributes to risk characterisation is also unsettled. ${ }^{16}$ The SPAF (stroke prevention in atrial fibrillation) study criteria have been shown reliably to identify low risk $\mathrm{AF}$ patients in two independent test cohorts. ${ }^{12}{ }^{17}$ The criteria from the atrial fibrillation investigators were derived from pooled analysis of participants in five trials, perhaps enhancing their generalisability. ${ }^{11}$ Validation of these schemes outside clinical trial cohorts is needed ${ }^{17}$ as community based cohorts are generally older and have a higher proportion of women. There is some evidence that existing risk stratification schemes may be age sensitive. ${ }^{15}$

$\mathrm{AF}$ patients categorised as having a moderate risk of stroke (about 3\% per year given aspirin) engender the most uncertainty about antithrombotic prophylaxis. Existing criteria for moderate risk are the most variable; it is unclear how reliably this subgroup can be characterised, the durability of classification, and the validity outside of clinical trials. Those doubting the reliability of currently available schemes to distinguish moderate from high risk generally advocate warfarin for all but the lowest risk patients.

Table 1 Magnitude of benefit according to inherent stroke risk in non-valvar atrial fibrillation

\begin{tabular}{|c|c|c|c|c|}
\hline & \multicolumn{3}{|c|}{ Primary prevention } & \multirow{2}{*}{$\begin{array}{l}\text { Secondary } \\
\text { prevention }\end{array}$} \\
\hline & Low & Moderate & High & \\
\hline Intrinsic rate (no treatment) & $1 \% /$ year & $3.5 \% /$ year & $6 \% /$ year & $12 \% /$ year \\
\hline \multicolumn{5}{|c|}{ Number of strokes prevented/year/1000 treated } \\
\hline Adjusted dose warfarin $v$ none & 6 & 21 & 36 & 84 \\
\hline Aspirin $v$ none & 2 & 7 & 12 & 12 \\
\hline Warfarin $v$ aspirin & 4 & 14 & 24 & 72 \\
\hline
\end{tabular}

General estimates to illustrate the effect of risk stratification on the magnitude of treatment effects. Relative risk reductions are $60 \%$ for warfarin $v$ none; $20 \%$ for aspirin $v$ none; and $40 \%$ for warfarin $v$ aspirin for primary prevention with corresponding reductions of $70 \%, 10 \%$, and $60 \%$ for secondary prevention. 
Table 2 Schemes for stratification of stroke risk in non-valvar atrial fibrillation

\begin{tabular}{|c|c|c|c|}
\hline & High risk & Moderate risk & Low risk \\
\hline \multicolumn{4}{|l|}{ Untreated patients } \\
\hline \multirow[t]{4}{*}{ Atrial Fibrillation Investigators $(1994)^{11}$} & Age $\geqslant 65$ years & & Age $<65$ years \\
\hline & History of hypertension & & No high risk features \\
\hline & Diabetes & & \\
\hline & Prior stroke/TIA & & \\
\hline Observed stroke rate & $5.1 \% /$ year & NA & $1.0 \% /$ year \\
\hline \multirow{4}{*}{$\begin{array}{l}\text { American College of Chest Physicians Consensus } \\
(1998)^{14}\end{array}$} & Age $>75$ years & Age $65-75$ years & Age $<65$ years \\
\hline & History of hypertension & Diabetes & No risk factors \\
\hline & Prior stroke/TIA & Coronary disease & \\
\hline & $\begin{array}{l}\text { Left ventricular dysfunction } \\
>1 \text { moderate risk factor }\end{array}$ & Thyrotoxicosis & \\
\hline Observed stroke rate & NA & NA & NA \\
\hline \multicolumn{4}{|l|}{ Aspirin treated patients } \\
\hline \multirow{4}{*}{ Stroke Prevention in Atrial Fibrillation $(1995)^{12}$} & Women $>75$ years & History of hypertension & No high risk features \\
\hline & Systolic BP > $160 \mathrm{~mm} \mathrm{Hg}$ & No high risk features & No history of hypertension \\
\hline & Prior stroke/TIA & & \\
\hline & Left ventricular dysfunction $\dagger$ & & \\
\hline Observed stroke rate & $7.7 \% /$ year & $3.6 \% /$ year & $1.1 \% /$ year \\
\hline \multirow[t]{4}{*}{ SPAF Exploratory Analysis $(1999)^{13}$} & Women $>75$ years & Hypertension and & No high or moderate risk features \\
\hline & Systolic $>160 \mathrm{~mm} \mathrm{Hg}$ & age $\leqslant 75$ years & \\
\hline & Hypertension plus age $>75$ & Diabetes & \\
\hline & Prior stroke/TIA & No high risk features & \\
\hline Observed stroke rate & $7.1 \% /$ year & $2.6 \% /$ year & $0.9 \% /$ year \\
\hline
\end{tabular}

${ }^{\star}$ Moderate to severe left ventricular systolic dysfunction on echocardiography or recent congestive heart failure.

$\dagger$ Impaired left ventricular function included recent congestive heart failure or fractional shortening $\leqslant 25 \%$ by $M$ mode echocardiography.

TIA, transient ischaemic attack.

Patient values and preferences after explanation of the benefits and risks of anticoagulation are particularly important in this risk stratum, although patient choices can be subtly - and not so subtly-influenced by physician perceived fears, both warranted and exaggerated, of anticoagulation.

\section{Appropriate use of warfarin in AF}

Considering the rapid pace of developments and lack of general consensus defining appropriate use, it is not surprising that many surveys have documented suboptimal use of warfarin, despite its potential to confer important benefits for many AF patients. Additional efforts to achieve consensus in this confusing area are needed. Additional data concerning prediction of stroke risk are required, including pathophysiological correlates and application of risk stratification schemes in clinical practice. Now that the efficacy and safety of warfarin and aspirin for prevention of stroke in AF patients have been reasonably well defined, ${ }^{1}$ reliable prediction of stroke risk to identify those who can benefit most and least from lifelong anticoagulation is presently the salient clinical issue, as emphasised by the two studies in this issue. ${ }^{45}$ All patients with AF should carefully be evaluated for factors conferring additional risk, and their stroke risk estimated. Those deemed high risk and many of those presumed at moderate risk should be considered for anticoagulation, regardless of age.

ROBERT G HART

Professor of Medicine (Neurology), University of Texas,

7703 Floyd Curl Drive, San Antonio, TX 78209, USA

1 Hart RG, Benavente $\mathrm{O}$, McBride R, et al. Antithrombotic therapies to prevent stroke in atrial fibrillation: a meta-analysis. Ann Intern Med 1999;131:492-501.
2 EAFT (European Atrial Fibrillation Trial) Study Group. European atrial fibrillation trial: secondary prevention in non-rheumatic atrial fibrillation after transient ischaemic attack or minor stroke. Lancet 1993;342: $1255-62$.

3 Kopecky SL, Gersh BJ, McGown MD, et al. The natural history of lone atrial fibrillation: a population-based study over three decades. $N$ Engl $\mathcal{F}$ Med 1987;317:669-74

4 Perez I, Melbourn A, Kalra L. Appropriateness of antithrombotic measures for stroke prevention in atrial fibrillation. Heart 1999;82:570-4.

5 Deplanque D, Corea F, Arquizan C, et al, and the SAFE I Study Investigators. Stroke and atrial fibrillation. Was stroke prevention appropriate beforehand? Heart 1999;82:563-9.

6 Stroke Prevention in Atrial Fibrillation Investigators. Bleeding during antithrombotic therapy in patients with atrial fibrillation. Arch Intern Med 1996;156:409-16.

7 Stroke Prevention in Atrial Fibrillation Investigators. Adjusted-dose warfarin versus low-intensity, fixed-dose warfarin plus aspirin for high-risk patients with atrial fibrillation: the stroke prevention in atrial fibrillation III randomized clinical trial. Lancet 1996;348:633-8.

8 Hylek EM, Skates SJ, Sheehan MA, et al. An analysis of the lowest effective intensity of prophylactic anticoagulation for patients with nonrheumatic atrial fibrillation. N Engl f Med 1996;335:540-6.

9 Hart RG. Intensity of anticoagulation to prevent stroke in patients with atrial fibrillation [letter]. Ann Intern Med 1998;128:408.

10 Sudlow M, Thomson R, Thwaites B, et al. Prevalence of atrial fibrillation and eligibility for anticoagulants in the community. Lancet 1998;352:116771.

11 Atrial Fibrillation Investigators. Risk factors for stroke and efficacy of antithrombotic therapy in atrial fibrillation: analysis of pooled data from five randomized controlled trials. Arch Intern Med 1994;154:1449-57.

12 The SPAF III Writing Committee for the Stroke Prevention in Atrial Fibrillation Investigators. Patients with nonvalvular atrial fibrillation at low risk of stroke during treatment with aspirin: stroke prevention in atrial fibrillation III study. $\mathcal{F} A M A$ 1998;279:1273-7.

13 Hart RG, Pearce LA, McBride R, et al, and the SPAF Investigators. Factors associated with ischemic stroke during aspirin therapy in atrial fibrillation. Stroke 1999;30:1223-9.

14 Laupacis A, Albers GW, Dalen JE, et al. Antithrombotic therapy in atrial fibrillation. Chest 1998;114(suppl):579-89S

15 Pearce LA, Hart RG, Halperin JL. Predicting stroke in atrial fibrillation: comparison of three risk stratification schemes. Am $\mathcal{F}$ Med. [In press.]

16 Stroke Prevention in Atrial Fibrillation Committee on Echocardiography. Transesophageal echocardiographic correlates of thromboembolism in high-risk patients with atrial fibrillation. Ann Intern Med 1998;128:639-47.

17 Feinberg WM, Kronmal RA, Newman AB, et al. Stroke risk in an elderly population with nonvalvular fibrillation: the cardiovascular health study. $\mathcal{F}$ Gen Intern Med 1999;14:56-9. 